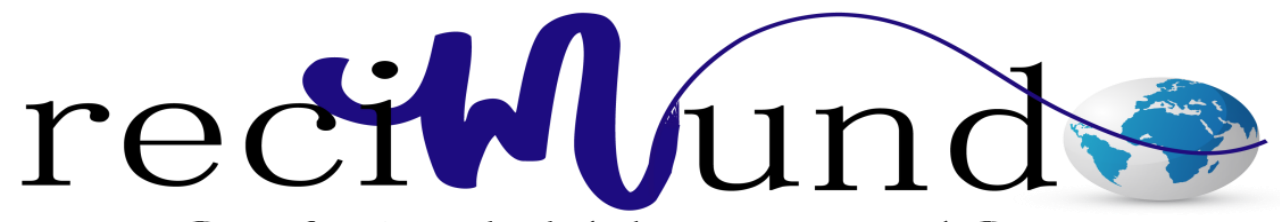

Revista Científica Mundo de la Investigación y el Conocimiento

Wilson Cando Tipan ${ }^{\text {a }}$; Josue Zumba Aguirre ${ }^{\text {b }}$ Jorge Bucheli ${ }^{\text {c }}$; Oscar Jaramillo ${ }^{\text {d }}$

Aplicación del Método Sónico con la Utilización del Equipo Pile Echo Tester para la Optimización del Factor de Seguridad de Pilotes. Caso de Estudio: Pilotes del Puente Peatonal de Muisne

Revista Científica Mundo de la Investigación y el Conocimiento. Vol. 1 núm., 5, diciembre, 2017, pp. 467-483

DOI: $10.26820 /$ recimundo/1.5.2017.467-483

Editorial Saberes del Conocimiento
a. Pontificia Universidad Católica del Ecuador; wocando@ puce.edu.com
b. Pontificia Universidad Católica del Ecuador; josue zumba@hotmail.com
c. Pontificia Universidad Católica del Ecuador; jabucheli@ puce.ed.ec
d. Pontificia Universidad Católica del Ecuador; ojaramillo602@puce.edu.ec 


\section{Aplicación del Método Sónico con la Utilización del Equipo Pile Echo Tester para la Optimización del Factor de Seguridad de Pilotes. Caso de Estudio: Pilotes del Puente Peatonal de Muisne}

Vol. 1, núm. 5., (2017)

Wilson Cando Tipan; Josue Zumba Aguirre; Jorge Bucheli; Oscar Jaramillo

\section{RESUMEN}

El presente artículo expone el principio del método sónico o ensayo de eco para la comprobación de la integridad estructural de pilotes que al ser una cimentación profunda es normalmente inaccesible en su totalidad, lo que impide o dificulta la verificación de que se haya ejecutado correctamente para asegurar que los pilotes de la cimentación de una estructura se comporten según lo previsto en el diseño. Se comienza definiendo los pilotes de hormigón armado construidos "in situ", su campo de aplicación, su normativa aplicable en el país y los posibles fallos que pueden presentar, para luego poder explicar la ejecución del método sónico y la forma de interpretación de los resultados a través de las anomalías en los reflectogramas obtenidos de los ensayos en los pilotes del Puente peatonal de Muisne, y asi poder determinar los posibles defectos físicos que pueden existir en el elemento. Finalmente, luego de realizada la interpretación de cada una de las curvas de Eco se determinara el factor de seguridad óptimo en el diseño de la cimentación si se realiza este tipo de ensayos para el control de calidad de los pilotes.

Palabras claves: Método sónico; integridad estructural; pilote; factor de seguridad; control de calidad. 


\title{
Aplicación del Método Sónico con la Utilización del Equipo Pile Echo Tester para la Optimización del Factor de Seguridad de Pilotes. Caso de Estudio: Pilotes del Puente Peatonal de Muisne
}

Vol. 1, núm. 5., (2017) Wilson Cando Tipan; Josue Zumba Aguirre; Jorge Bucheli; Oscar Jaramillo

\begin{abstract}
This document sets out the principle of the sonic echo pile test for the verification of the structural integrity of piles. The piles like deep foundations are normally inaccessible in their entirety, which makes it difficult to verify that it has been built correctly and therefore to be able to guarantee an adequate behavior of the pile according to the requirements required in the design. This document begins by defining the reinforced concrete piles built "in situ", its field of application, its regulations in the country and possible failures that may arise, and then be able to explain the execution of the sonic eco pile test and the way of interpretation of the results through the anomalies in the reflectograms obtained from the tests on the piles of the Muisne Pedestrian Bridge, so as to be able to determine the possible physical defects that may exist in them. Finally, after the interpretation of each one of the curves of Echo, the optimum safety factor in the design of the foundation will be determined in this document as long as this test is performed for the quality control of the piles.
\end{abstract}

Keywords: Sonic test; structural integrity; pile; safety factor; quality control. 


\section{Aplicación del Método Sónico con la Utilización del Equipo Pile Echo Tester para la Optimización del Factor de Seguridad de Pilotes. Caso de Estudio: Pilotes del Puente Peatonal de Muisne}

Vol. 1, núm. 5., (2017)

Wilson Cando Tipan; Josue Zumba Aguirre; Jorge Bucheli; Oscar Jaramillo

\section{Introducción.}

Los pilotes son sensibles a modos de ejecución deficientes especialmente durante la etapa de perforación y de hormigonado del pilote. En la mayoría de los tipos de pilotes todo el proceso constructivo se hace de modo oculto por lo que el producto final es generalmente invisible. Los ensayos de integridad estructural en pilotes proporcionan datos sobre la continuidad a lo largo del fuste, la homogeneidad del hormigón, la consistencia de los materiales empleados para su fabricación y sus dimensiones físicas, es decir, proporcionan una evaluación cualitativa del pilote y una verificación de su resistencia mecánica. (American Society of Civil Engineers, 1997) (Arnau Rodriguez, Fernandez Tadeo, \& Correa Llodera, 1993)

Este tipo de ensayos no reemplazan a los ensayos estáticos de carga en pilotes solo lo hacen en obras de poco presupuesto, puesto que no brindan información directa sobre su comportamiento en condiciones de carga pero proporcionan información rápida y económica de la presencia de defectos en los pilotes, lo que permite realizar un control de calidad de los mismos y ajustarse a un diseño más óptimo de la cimentación tal como lo permiten las normas internacionales cuando se realiza este tipo de ensayos. (Clyde N \& Elliote, 2010)

\section{Metodología.}

En el ensayo sónico de integridad estructural de pilotes realizado para nuestro caso de estudio utilizamos un equipo desarrollado por la empresa inglesa Piletest conocido como Pile Echo Tester (PET), realizándose el ensayo según los requerimientos establecidos en la Norma ASTM D5882-16 para ensayos de baja deformación de integridad estructural de pilotes. 


\section{Aplicación del Método Sónico con la Utilización del Equipo Pile Echo Tester para la Optimización del Factor de Seguridad de Pilotes. Caso de Estudio: Pilotes del Puente Peatonal de Muisne}

Vol. 1, núm. 5., (2017)

Wilson Cando Tipan; Josue Zumba Aguirre; Jorge Bucheli; Oscar Jaramillo

Debido a que el porcentaje estadístico de pilotes con presencia de fallos es reducido se decidió ensayar el 100\% de los pilotes y se estableció realizar tres ensayos en cada uno de ellos, lo que permite garantizar una mayor confiabilidad de los resultados obtenidos. Si los ensayos en un mismo pilote no son repetibles, no se deberán utilizar los datos para la interpretación y será necesario preparar el pilote para poder alcanzar las condiciones de repetibilidad.

El ensayo se realiza con un martillo de mano con el cual se da un golpe en el centro de la cabeza del pilote generando una onda de compresión que recorre a lo largo del fuste y es reflejada por la punta del pilote, por la presencia de discontinuidades en el fuste, por cambios en su sección transversal o por cambios de rigidez entre los estratos del terreno circundante, para ser captada por un acelerómetro que se encuentra en la cabeza del elemento y posteriormente ser transformada por un software en medida de velocidad en función del tiempo (reflectograma).

El análisis será cualitativo por comparación con otros pilotes ensayados anteriormente y en función del criterio del ingeniero que interpreta los resultados. La evaluación de los resultados obtenidos indicara que el pilote es aceptable, si no se presentan anomalías en las gráficas por lo que se puede asegurar que existe concordancia entre su comportamiento real y su comportamiento teórico; es defectuoso, si se concluye que los defectos afectan la capacidad estructural del mismo y será necesario establecer si el tipo de fallos que presenta puede ser solucionado o necesariamente el pilote debe ser reemplazado; y es cuestionable, si resulta complejo la interpretación de las gráficas obtenidas por lo que se deberá realizar ensayos 


\section{Aplicación del Método Sónico con la Utilización del Equipo Pile Echo Tester para la Optimización del Factor de Seguridad de Pilotes. Caso de Estudio: Pilotes del Puente Peatonal de Muisne}

Vol. 1, núm. 5., (2017)

Wilson Cando Tipan; Josue Zumba Aguirre; Jorge Bucheli; Oscar Jaramillo

complementarios como el ensayo cross-hole ultrasónico, o pruebas estáticas o dinámicas de carga.

En un reflectograma ideal de un pilote continuo con empotramiento libre la curva inicial y final tendrán la misma polaridad, lo mismo sucede en el caso de un estrechamiento del pilote puesto que existe una disminución de la sección transversal por donde recorren las ondas. Por otro lado, en un reflectograma ideal de un pilote continuo empotrado en roca o suelo duro la curva final tendrá distinta polaridad a la curva inicial, lo mismo ocurre en el caso de un ensanchamiento del pilote puesto que existe un aumento de la sección transversal por donde se desplazan las ondas.

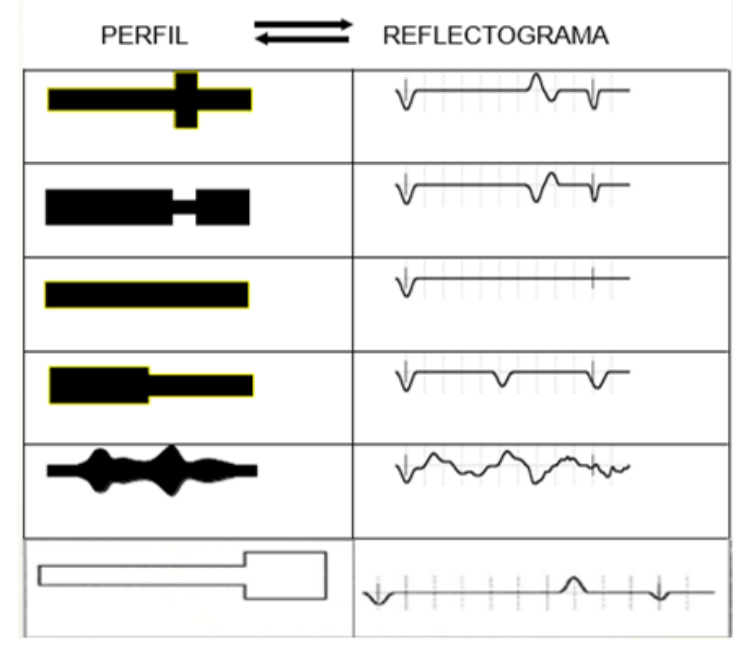

Figura 1. Interpretación de curvas teóricas de eco

Para determinar el factor de seguridad con la mayor probabilidad de falla se ha establecido la utilización del Método PERT o Método de los tres valores, debido a que presenta 


\section{Aplicación del Método Sónico con la Utilización del Equipo Pile Echo Tester para la Optimización del Factor de Seguridad de Pilotes. Caso de Estudio: Pilotes del Puente Peatonal de Muisne}

Vol. 1, núm. 5., (2017) Wilson Cando Tipan; Josue Zumba Aguirre; Jorge Bucheli; Oscar Jaramillo

una distribución beta triangular con una probabilidad del $50 \%$ obtenida a partir de tres datos: el valor pesimista, el valor más probable y el valor optimista.

$$
F S=\frac{P+4 M P+O}{6}
$$

Para nuestro caso de estudio se ha escogido los valores referenciales que permiten las normativas internacionales al reducirse las incertidumbres ocultas en el diseño y ejecución de la cimentación, debido a que la Norma Ecuatoriana de la Construcción no especifica los valores a utilizarse en el caso de realizarse este tipo de ensayos.

Para poder intuir las causas que produjeron el defecto físico en el pilote, se debe conocer el perfil geotécnico de su entorno, su proceso constructivo y posibles incidencias durante la perforación, durante el suministro de hormigón y durante el proceso de hormigonado, por tal razón, se ha establecido el siguiente formato para recopilar la información necesaria que permita una adecuada interpretación de los resultados obtenidos a través de estos ensayos. 
Aplicación del Método Sónico con la Utilización del Equipo Pile Echo Tester para la Optimización del Factor de Seguridad de Pilotes. Caso de Estudio: Pilotes del Puente Peatonal de Muisne

Vol. 1, núm. 5., (2017)

Wilson Cando Tipan; Josue Zumba Aguirre; Jorge Bucheli; Oscar Jaramillo

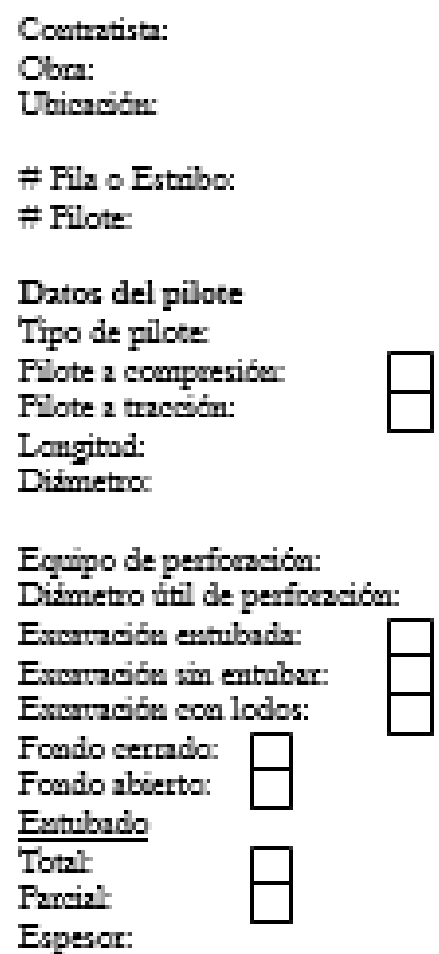

Espesor

\section{Método oonstructivox}

Amadura.

Longitod:

Colocasion de la ammadun Antes del hormigonadox

Despues del bormigoudio: Estribo

Tipo:

Diemetroc

Esprciumientoc

\section{Hormigón}

Resistencia a la comptevidu:

Consistenciz (Cono de Abrums):

Empleo de vibudor:

Contenido de cementox

Trmuio maximo de iridos:

Relacion agua / cemento:

Consumo de borrsigón

Técies:

Real:
Aclitivos en el bocrmigón:

$\%$ del pero del cemento:

Tiempo de trabziabilidad:

Colosasion del bormigón

Sumergidox

En seco $\alpha$

Tubo Tremie:

Dimotro interior:

Limpiera de la base del pilate:

Tiempos de ejecución

\begin{tabular}{|l|l|l|l|}
\hline \multicolumn{1}{|c|}{ Proceso } & Hora inicio & Hora in & Fecha \\
\hline Excurzobu & & & \\
\hline $\begin{array}{l}\text { Colocadion de } \\
\text { armudara }\end{array}$ & & & \\
\hline Homigonzdo & & & \\
\hline
\end{tabular}

Corte estratigráfico

\begin{tabular}{|l|l|l|l|}
\hline Frofundidad & SUCS & NEC & Nivel freático \\
\hline & & & \\
\hline & & & \\
\hline & & & \\
\hline & & & \\
\hline & & & \\
\hline & & & \\
\hline & & & \\
\hline & & & \\
\hline & & & \\
\hline & & & \\
\hline & & & \\
\hline & & & \\
\hline & & & \\
\hline & & & \\
\hline & & & \\
\hline & & & \\
\hline & & & \\
\hline & & & \\
\hline
\end{tabular}

Comentarios y observaciones: 
Aplicación del Método Sónico con la Utilización del Equipo Pile Echo Tester para la Optimización del Factor de Seguridad de Pilotes. Caso de Estudio:

Pilotes del Puente Peatonal de Muisne

Vol. 1, núm. 5., (2017)

Wilson Cando Tipan; Josue Zumba Aguirre; Jorge Bucheli; Oscar Jaramillo

Resultados y Discusión.

Edad del hormigón al momento del ensayo: 5 dias

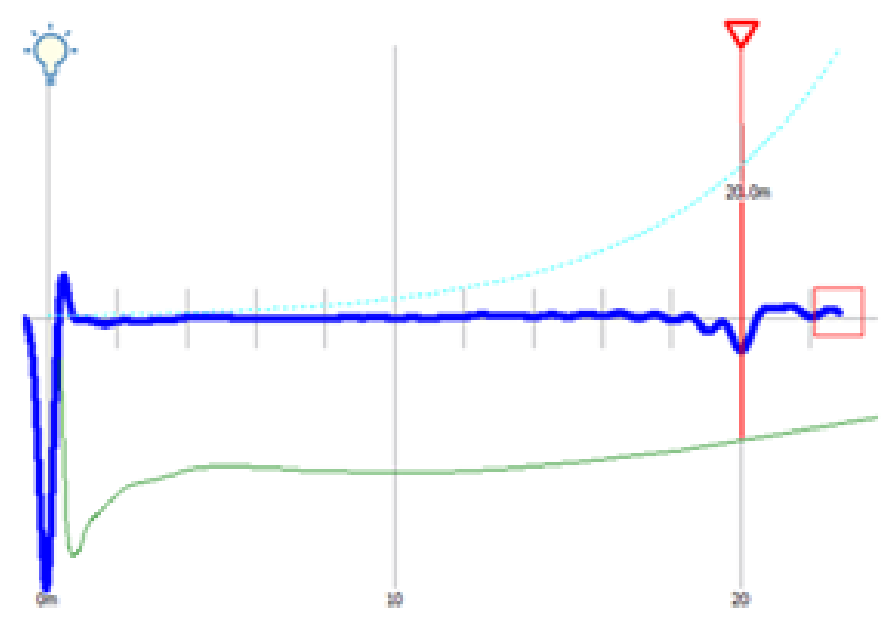

Figura 2. Reflectograma del pilote 4 del estribo 1

Edad del hormigón al momento del ensayo: 7 dias

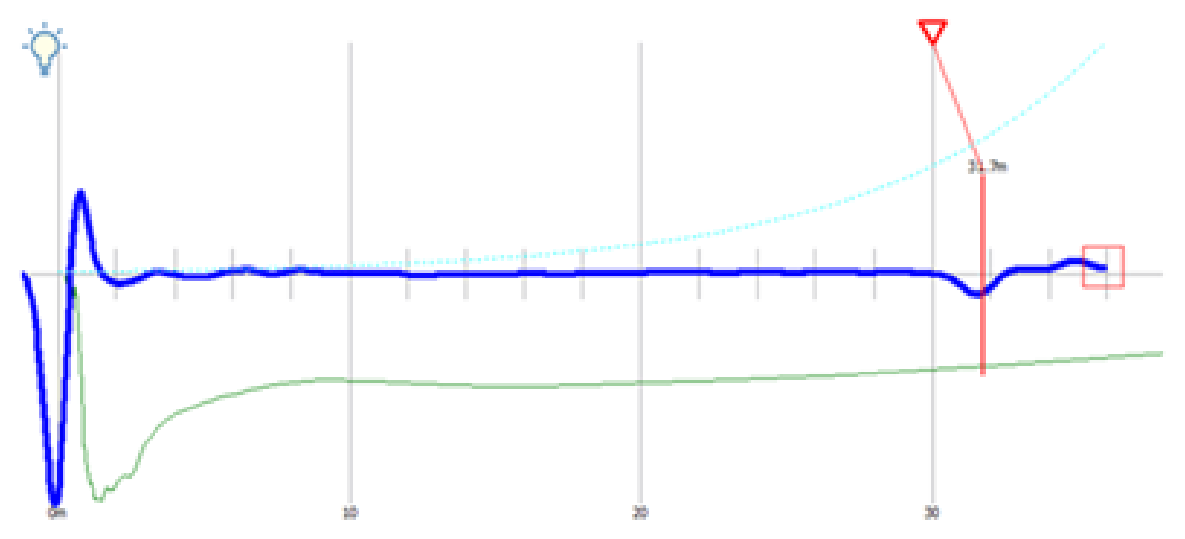

Figura 3. Reflectograma del pilote 2 de la pila 1 
Aplicación del Método Sónico con la Utilización del Equipo Pile Echo Tester para la Optimización del Factor de Seguridad de Pilotes. Caso de Estudio: Pilotes del Puente Peatonal de Muisne

Vol. 1, núm. 5., (2017)

Wilson Cando Tipan; Josue Zumba Aguirre; Jorge Bucheli; Oscar Jaramillo

Edad del hormigón al momento del ensayo: 10 días

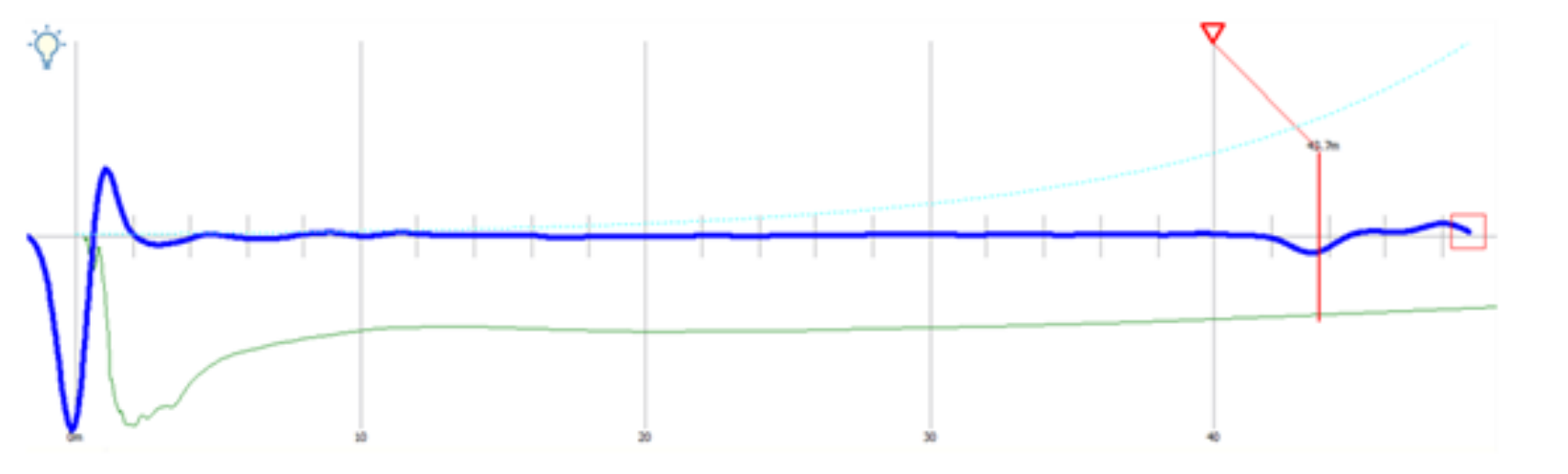

Figura 4. Reflectograma del pilote 3 de la pila 2

Con base en los resultados de los ensayos de integridad estructural realizados en los pilotes que conforman los estribos y pilas del Puente Peatonal de Muisne, se evidencia que no existe presencia de anomalías en los reflectogramas, lo que indica la existencia de pilotes integros sin heterogeneidades con una longitud similar a la esperada y cuya punta esta empotrada en roca o suelo duro (lutita).

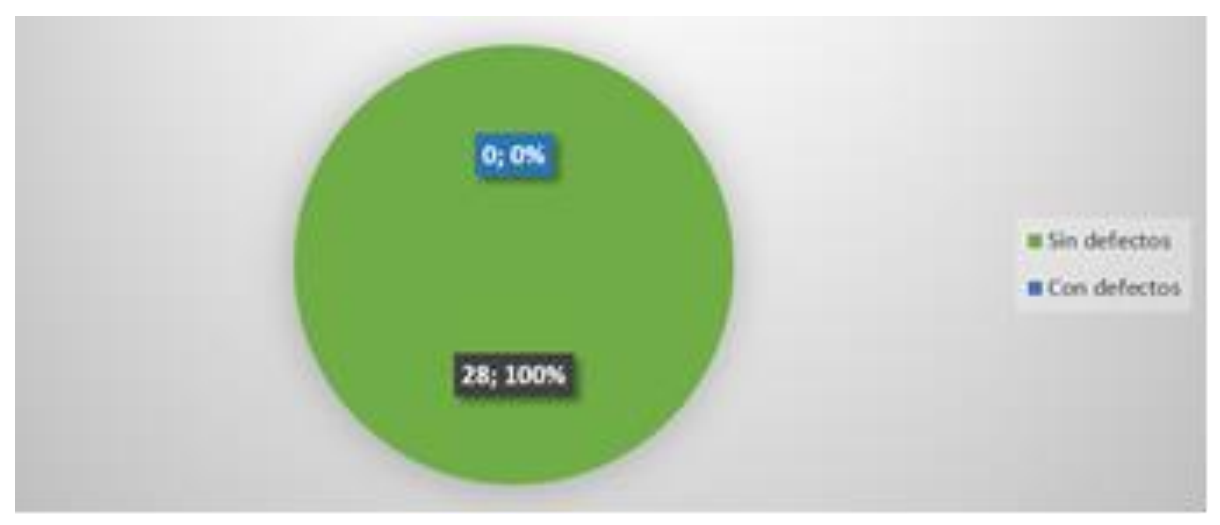

Figura 5. Resultados de los ensayos de integridad estructural en los pilotes del Puente peatonal de Muisne. 


\section{Aplicación del Método Sónico con la Utilización del Equipo Pile Echo Tester}

para la Optimización del Factor de Seguridad de Pilotes. Caso de Estudio: Pilotes del Puente Peatonal de Muisne

Vol. 1, núm. 5., (2017) Wilson Cando Tipan; Josue Zumba Aguirre; Jorge Bucheli; Oscar Jaramillo

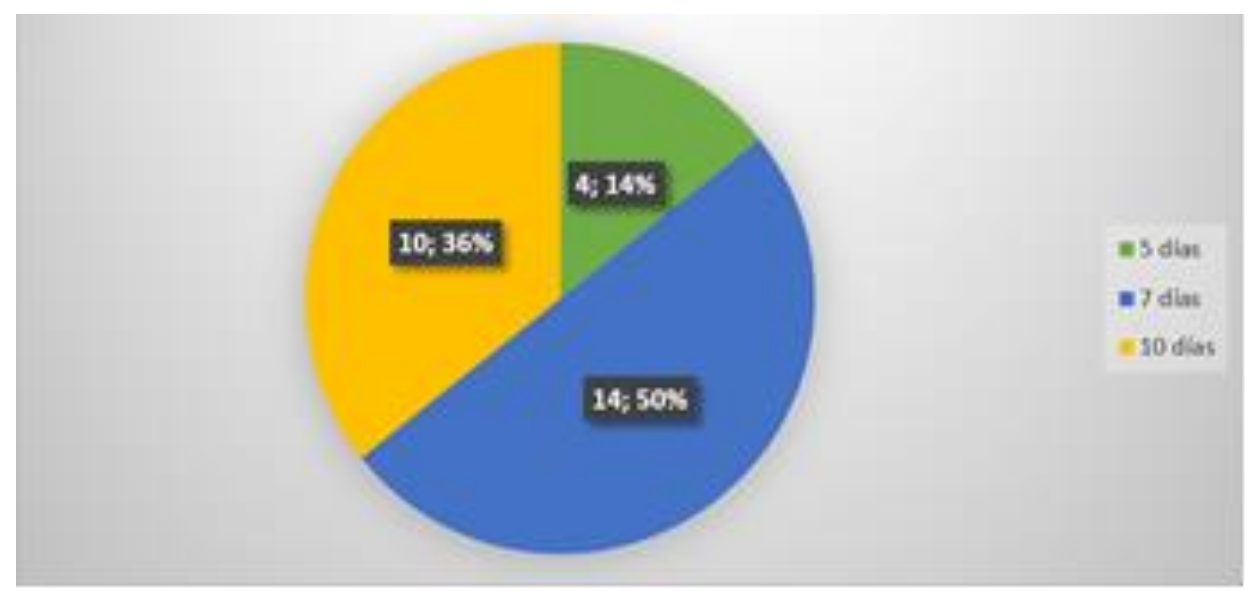

Figura 6. Número de pilotes ensayados según la edad del hormigón al momento del ensayo

Diversos autores recomiendan que para la realización del ensayo el pilote debe haber alcanzado una edad mínima de siete días de manera que se alcance el grado de endurecimiento del hormigón que permita garantizar que la onda se propague a lo largo del fuste, aunque en nuestro estudio realizamos ensayos a edades de cinco, siete y diez días, alcanzando resultados muy satisfactorios a edades más tempranas que la recomendada.

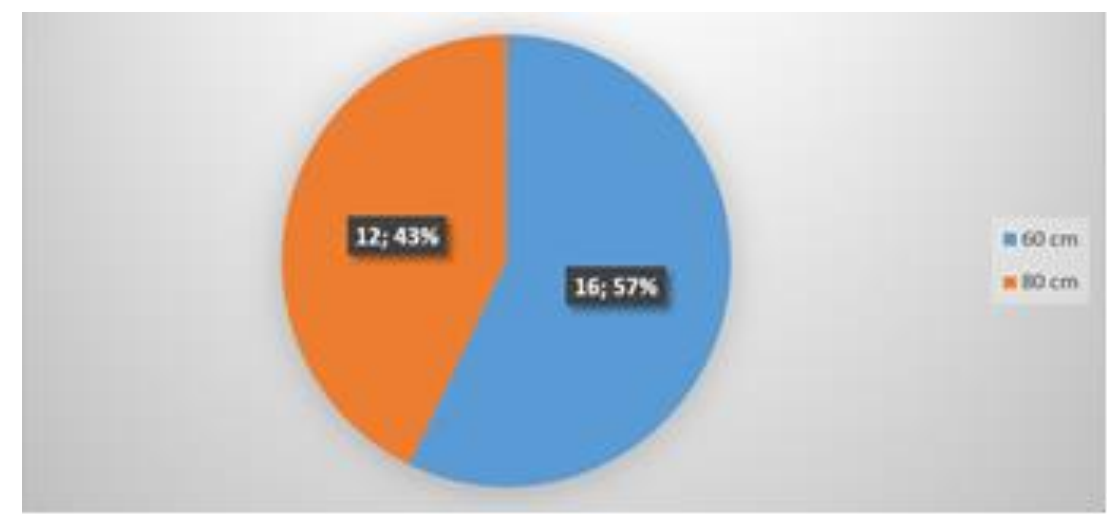

Figura 7. Número de pilotes ensayados según la medida de su diámetro 


\section{Aplicación del Método Sónico con la Utilización del Equipo Pile Echo Tester para la Optimización del Factor de Seguridad de Pilotes. Caso de Estudio: Pilotes del Puente Peatonal de Muisne}

Vol. 1, núm. 5., (2017)

Wilson Cando Tipan; Josue Zumba Aguirre; Jorge Bucheli; Oscar Jaramillo

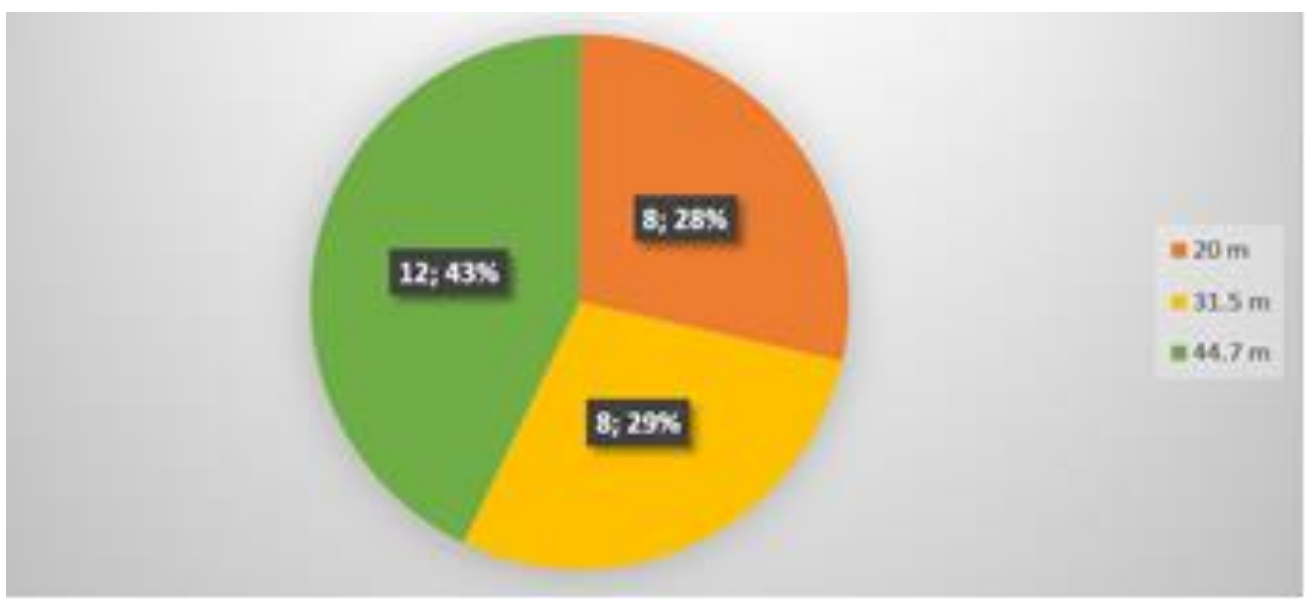

Figura 8. Número de pilotes ensayados según la medida de su longitud

Como se puede observar el ensayo no tiene limitaciones en cuanto a la longitud o diámetro del pilote, pero existen limitantes en lo que se refiere a la identificación de desvíos en la armadura que compone el pilote, en la comprobación de la verticalidad del elemento, en el análisis de la posición lateral de los defectos (si están en el centro o en la periferia) y entrega resultados distorsionados para elementos bidireccionales como las pantallas de hormigón. Realmente, incluso con estas limitaciones, son una buena herramienta para detectar defectos significativos y comprobar la longitud teórica de los pilotes de forma sencilla.

La selección del valor del factor de seguridad es bastante subjetiva debido a que depende del tamaño e importancia de la obra, de la calidad de la información geotécnica y de las consecuencias de un posible fallo en la estructura o en la cimentación.

Es posible determinar valores máximos de factores de seguridad para la condición más desfavorable y valores mínimos de factores de seguridad para la condición más favorable, lo que 


\section{Aplicación del Método Sónico con la Utilización del Equipo Pile Echo Tester para la Optimización del Factor de Seguridad de Pilotes. Caso de Estudio: Pilotes del Puente Peatonal de Muisne}

Vol. 1, núm. 5., (2017) Wilson Cando Tipan; Josue Zumba Aguirre; Jorge Bucheli; Oscar Jaramillo

permitirá obtener mediante un análisis probabilístico el valor de factor de seguridad con la mayor probabilidad de falla.

Para determinar el factor de seguridad con la mayor probabilidad de falla se ha establecido la utilización del Método PERT o Método de los tres valores: el valor pesimista, el valor más probable y el valor optimista, debido a que la Norma Ecuatoriana de la Construcción no especifica los valores a utilizarse en el caso de realizarse este tipo de ensayos.

En el diseño de la cimentación del Puente Peatonal de Muisne se utilizó el factor de seguridad de 3 al cual lo vamos a considerar nuestro valor pesimista, mismo que recomienda nuestra normativa y engloba una serie de incertidumbres que entrañan las cimentaciones profundas.

El valor más probable será el que estipula la Normativa francesa sobre pilotes (DTU 13.2) y el capítulo de Recomendaciones para Obras Marítimas (ROM 0.5-05) de la Normativa Española de Proyectos Portuarios, donde se señala que si se realizan este tipo de ensayos se podrá incrementar las cargas admisibles de los pilotes por razón estructural en un 20\%, lo que resultaría para nuestro caso de estudio al considerar en los diseños un valor referencial de 3 , en un valor del factor de seguridad de 2.50 .

$$
F S=\frac{3+(4 \times 2.5)+2.4}{6}=2.57 \approx 2.60
$$




\section{Aplicación del Método Sónico con la Utilización del Equipo Pile Echo Tester para la Optimización del Factor de Seguridad de Pilotes. Caso de Estudio: Pilotes del Puente Peatonal de Muisne}

Vol. 1, núm. 5., (2017)

Wilson Cando Tipan; Josue Zumba Aguirre; Jorge Bucheli; Oscar Jaramillo

Finalmente, el análisis probabilístico realizado utilizando el Método PERT o Método de los tres valores permitió determinar el valor de 2.60 para el factor de seguridad en el diseño de la cimentación del Puente peatonal de Muisne, siempre que los ensayos de integridad estructural realizados en la totalidad de los pilotes confirmen la calidad de los mismos, es decir, siempre que estos no presenten defectos.

El valor más optimista será el que establece el Código Técnico de la Edificación donde se sugiere que si se realizan ensayos de integridad estructural en la totalidad de los pilotes o en una muestra significativa, las cargas admisibles por razón estructural en el diseño de los pilotes se podrán incrementar en un $25 \%$, lo que resultaría para nuestro caso de estudio en un valor del factor de seguridad de 2.40 .

\section{Conclusiones.}

El ensayo de integridad debe ser un elemento de inspección que si es necesario se tendrá que complementar con investigaciones complementarias que se indicarán en el informe, por lo que no es un árbitro final para decir si está bien o mal un pilote.

Los ensayos de integridad estructural reducen o eliminan las incertidumbres propias de las cimentaciones profundas, permitiendo reducir los factores de seguridad utilizados en el diseño y consecuentemente reducir el costo de la cimentación. 


\section{Aplicación del Método Sónico con la Utilización del Equipo Pile Echo Tester para la Optimización del Factor de Seguridad de Pilotes. Caso de Estudio: Pilotes del Puente Peatonal de Muisne}

Vol. 1, núm. 5., (2017) Wilson Cando Tipan; Josue Zumba Aguirre; Jorge Bucheli; Oscar Jaramillo

La punta del pilote no podría ser observada claramente en la curva del Eco si los pilotes están empotrados en una roca compacta que tenga características similares de impedancia acústica como el hormigón, debido a que no existiría cambio de rigidez entre los dos materiales.

Generalmente se piensa que si se realiza la excavación y el hormigonado con camisa perdida, los pilotes estarán exentos de defectos; esto no es del todo cierto, debido a que pueden existir problemas en la calidad del hormigón, pero de lo que sí se puede estar seguro es de que reducen significativamente los riesgos de la presencia de defectos en los pilotes, tal como se evidencia en los pilotes del puente peatonal de Muisne que no han presentado anomalías en los reflectogramas, debido en parte a que han sido ejecutados con camisa recuperable, pero sobre todo a la calidad del hormigón.

De los resultados no se puede extraer directamente que pequeñas variaciones de impedancia serán fatales para el comportamiento del pilote. El ingeniero deberá juzgar la aceptabilidad de los pilotes con heterogeneidades, considerando otros factores como la redistribución de cargas en pilotes adyacentes, transferencias de cargas al terreno por encima de la heterogeneidad, coeficientes de seguridad adoptados y requerimientos reales de carga de la estructura sobre los pilotes.

Establecer un análisis totalmente automatizado de los resultados de estos ensayos resultaría bastante complejo, debido al gran número de causas que pueden provocar variaciones de impedancia. 


\section{Aplicación del Método Sónico con la Utilización del Equipo Pile Echo Tester para la Optimización del Factor de Seguridad de Pilotes. Caso de Estudio: Pilotes del Puente Peatonal de Muisne}

Vol. 1, núm. 5., (2017)

Wilson Cando Tipan; Josue Zumba Aguirre; Jorge Bucheli; Oscar Jaramillo

El ensayo de Eco permite analizar si el elemento se encuentra en una situación de empotramiento en punta o de pilote libre, por lo que se pudo confirmar que los pilotes de las pilas centrales del Puente peatonal de Muisne se encuentran empotrados en roca o suelo duro (lutita).

El análisis probabilístico realizado utilizando el Método PERT o Método de los tres valores permitió determinar el valor de 2.60 para el factor de seguridad en el diseño de la cimentación del puente peatonal de Muisne, siempre que los ensayos de integridad estructural se realicen en la totalidad de los pilotes para confirmar la calidad de los mismos, es decir, para verificar que no existan defectos en los elementos.

Hasta la fecha no existe en Ecuador ningún documento normativo que regule la ejecución e interpretación de estos ensayos, a pesar de que permiten verificar la continuidad del fuste y la resistencia mecánica del hormigón.

Se recomienda que el constructor proporcione todos los datos requeridos en el formato propuesto, para realizar una adecuada interpretación de los resultados obtenidos y establecer las posibles causas que provocaron los defectos detectados.

No se recomienda utilizar esta técnica para obtener información sobre la capacidad portante del pilote, ya que sería arriesgado obtener conclusiones sobre este aspecto. 


\section{Aplicación del Método Sónico con la Utilización del Equipo Pile Echo Tester para la Optimización del Factor de Seguridad de Pilotes. Caso de Estudio: Pilotes del Puente Peatonal de Muisne \\ Vol. 1, núm. 5., (2017) Wilson Cando Tipan; Josue Zumba Aguirre; Jorge Bucheli; Oscar Jaramillo}

\section{Bibliografía.}

American Society of Civil Engineers. (1997). Standard guidelines for the design and installation of pile foundations. New York: ASCE standard.

Arnau Rodriguez, J., Fernandez Tadeo, C., \& Correa Llodera, J. (1993). Ensayo de control de integridad en pilotes. Buenos Aires: Cimbra.

Clyde N, B., \& Elliote, D. (2010). Use of Nondestructive Testing To Evaluate Defects in Drilled Shafts: Results of FHW A Research. Transportation Research Record 1331 , 28-35. 